\title{
Photocurrent and photoconductance properties of a GaAs nanowire
}

\author{
S. Thunich, ${ }^{1}$ L. Prechtel, ${ }^{1}$ D. Spirkoska, ${ }^{1}$ G. Abstreiter, ${ }^{1}$ A. Fontcuberta i Morral, ${ }^{1,2}$ and \\ A. W. Holleitner ${ }^{1, a)}$ \\ ${ }^{1}$ Physik Department and Walter Schottky Institut, Technische Universität München, Am Coulombwall 3, \\ D-85748 Garching, Germany \\ ${ }^{2}$ Laboratoire des Matériaux Semiconducteurs, Institut des Matériaux, Ecole Polytechnique Fédérale de \\ Lausanne, CH-1015 Lausanne, Switzerland
}

(Received 19 May 2009; accepted 11 July 2009; published online 26 August 2009)

\begin{abstract}
We report on photocurrent and photoconductance processes in a freely suspended $p$-doped single GaAs nanowire. The nanowires are grown by molecular beam epitaxy, and they are electrically contacted by a focused ion beam deposition technique. The observed photocurrent is generated at the Schottky contacts between the nanowire and metal source-drain electrodes, while the observed photoconductance signal can be explained by a photogating effect induced by optically generated charge carriers located at the surface of the nanowire. Both optoelectronic effects are sensitive to the polarization of the exciting laser field, enabling polarization dependent photodetectors. (C) 2009 American Institute of Physics. [DOI: 10.1063/1.3193540]
\end{abstract}

Semiconductor nanowires have attracted considerable attention for the past few years because of their compelling electronic, mechanical, and optical properties. ${ }^{1-10}$ A very suitable and versatile technique for nanowire growth is the direct synthesis on a substrate. ${ }^{11-14}$ The fabrication of III-V semiconductor nanowire based devices by such a bottom-up approach ensures the rational use of materials, as the nanowires can be obtained in principle on any substrate. Here, we report on the optoelectronic properties of photodetectors based on single $p$-doped GaAs nanowires grown by molecular beam epitaxy (MBE) with the so-called vapor-liquid-solid method using $\mathrm{Ga}$ droplets as self-catalysts. ${ }^{15-17}$ The nanowires are electrically contacted by metal electrodes using a focused ion beam (FIB) deposition technique. ${ }^{18,19}$ We experimentally identify two dominating optoelectronic processes in the metal-GaAs nanowire-metal photodetectors. On the one hand, there is a photocurrent generated at the Schottky contacts between the GaAs nanowires and the metal sourcedrain electrodes, as recently shown for $\mathrm{CdS}$ nanowires. ${ }^{8}$ On the other hand, we observe a photoconductance effect, when illuminating the GaAs nanowire far away from the contacts. We interpret the photoconductance effect to arise from band bending effects caused by surface states on the nanowire surface. In particular, optically generated excess electrons are trapped at the surface, where they act as a negative gating voltage on the $p$-doped nanowires (photogating effect). At the same time, the optically excited free excess holes raise the Fermi energy of the hole gas within the nanowires (photodoping effect). Both effects can raise the conductance of the semiconductor circuits. ${ }^{20,21}$ We demonstrate that both photocurrent and photoconductance effects are sensitive to the orientation of linear polarized light. The photoconductance (-current) varies by $\sim 35 \%(\sim 15 \%)$ for the photon polarization being parallel or perpendicular to the direction of the GaAs nanowires. Hereby, the metal-GaAs nanowiremetal circuits act as polarization-sensitive photodetectors, ${ }^{2}$

\footnotetext{
a) Author to whom correspondence should be addressed. Electronic mail: holleitner@wsi.tum.de.
}

which can be integrated into electronic circuits by the FIBdeposition technique in a very versatile way.

Starting point are GaAs nanowires, which are grown by $\mathrm{MBE}$ on a $\mathrm{SiO}_{2}$ covered (111)-oriented GaAs substrate as presented previously (growth rate of $\sim 0.25 \AA / s, \mathrm{As}_{4}$ partial pressure of $\sim 2 \times 10^{-6}$ mbar, growth temperature of $\sim 630{ }^{\circ} \mathrm{C}$, and $7 \mathrm{rpm}$ rotation). ${ }^{17}$ The nanowires with $\{110\}$ facets have a length (diameter) of $\sim 10 \mu \mathrm{m}(\sim 140 \mathrm{~nm})$, and they are doped type $p$ with a carrier concentration of $\sim 10^{18} \mathrm{~cm}^{-3}$ by adding a silicon flux during the growth. ${ }^{17}$ The scanning electron micrograph (SEM) image in Fig. 1(a) shows the photodetector circuit with such a $p$-type GaAs nanowire bridging two gold pads acting as "source" and "drain" electrodes. The pads are first evaporated on top of a $\mathrm{SiO}_{2}$ chip utilizing a shadow mask, and they act as sourcedrain electrodes for the nanowire. The $\mathrm{SiO}_{2}$ substrate in between the pads is milled away to a depth of a few hundred nanometers by the help of a FIB microscope. ${ }^{18}$ Following the
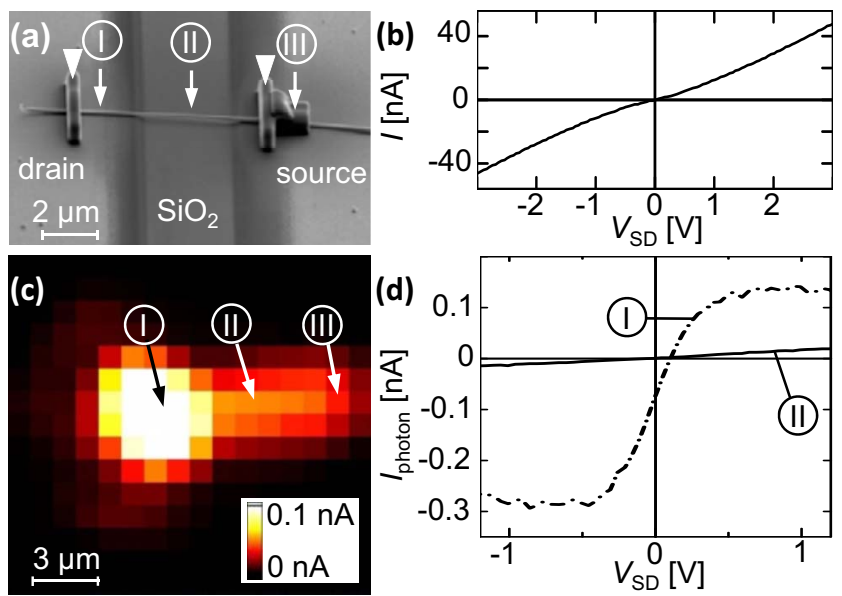

FIG. 1. (Color online) (a) SEM image of esa freely suspended $p$-doped GaAs nanowire bridging two gold electrodes (sample A). (b) $I-V$ characteristics of sample A without laser excitation. (c) Photocurrent contour plot of $\left|I_{\text {photon }}\right|$ as a function of the spatial coordinates of sample A $\left(T_{\text {bath }} \sim 296 \mathrm{~K}\right.$, $I_{\text {laser }} \sim 60 \mathrm{~W} / \mathrm{cm}^{2}, f_{\text {chop }}=1028 \mathrm{~Hz}, \lambda=780 \mathrm{~nm}$, and $\left.V_{\mathrm{SD}}=+1 \mathrm{~V}\right)$. (d) $I_{\text {photon }}$ as function of $V_{\mathrm{SD}}$ for positions I and II of sample A $\left(T_{\text {bath }} \sim 296 \mathrm{~K}, I_{\text {lase }}\right.$ $\sim 30 \mathrm{~W} / \mathrm{cm}^{2}, f_{\text {chop }}=930 \mathrm{~Hz}$, and $\lambda=730 \mathrm{~nm}$ ). 
technique described in Ref. 19, the particular nanowire is positioned at the shown location by using mechanical manipulators within the FIB recipient. The nanowire is attached to the source-drain electrodes by a FIB induced deposition of carbon utilizing phenanthren $\left[\mathrm{C}_{14} \mathrm{H}_{10}\right]$ as a precursor gas [triangles in Fig. 1(a)]. The circuit in Fig. 1(a) features three positions: I, II, and III. At positions I and III, Schottky contacts between the nanowire and the gold electrodes can be assumed. At position III, however, the nanowire is covered by an opaque carbon layer with a thickness of $\sim 350 \mathrm{~nm}$. At position II, the nanowire is freely suspended. Hereby, the circuit allows locally addressing the optoelectronic processes in the middle of the nanowires (position II) independently from the ones occurring at the metal-semiconductor contact (position I).

We present optoelectronic measurements of two independent samples $\mathrm{A}$ and $\mathrm{B}$, which are typical for the measured $p$-doped nanowires. The samples are mounted in a vacuum chamber at a pressure of about $10^{-5}$ mbar. Figure 1(b) depicts the typical $I-V$ characteristics of the devices. Since the $I-V$-characteristics are nonlinear and symmetric with respect to the bias voltage, we infer that FIB-fabricated samples act as metal-semiconductor-metal devices with equivalent Schottky barriers at each end of the nanowires. ${ }^{22}$ The possible morphology of the Schottky contacts with the additional carbon deposits close by will be discussed elsewhere. Most importantly, the reported optoelectronic findings only rely on the presence of back-to-back Schottky barriers with a $p$-doped GaAs nanowire in between. Optical excitation occurs by focusing the light of a mode-locked titanium:sapphire laser with a repetition rate of $76 \mathrm{MHz}$ through the objective of a microscope onto the nanowire circuits. The laser can be tuned in the energy range of $1.24 \mathrm{eV}<E_{\text {photon }}$ $<1.77 \mathrm{eV}$. With a spot diameter of $\sim 2 \mu \mathrm{m}$ the light intensity $I_{\text {laser }}$ is of the order of $100 \mathrm{~W} / \mathrm{cm}^{2}$ for all $E_{\text {photon }}$. Generally, we chop the laser at a frequency $f_{\text {chop. }}$. For the optoelectronic measurements discussed below, the resulting current $I_{\text {photon }}=I_{\text {on }}\left(\lambda_{\text {photon }}, f_{\text {chop }}\right)-I_{\text {off }}\left(\lambda_{\text {photon }}, f_{\text {chop }}\right)$ across the sample with the laser being "on" or "off," respectively, is amplified by a current-voltage converter and detected with a lock-in amplifier utilizing the reference signal provided by the chopper. ${ }^{21}$

We acquire photocurrent images of the nanowire circuits by recording the change of $I_{\text {photon }}$ at a finite source-drain bias $V_{\mathrm{SD}}$, when the laser spot is laterally scanned across the samples. ${ }^{20}$ Then, $I_{\text {photon }}=I_{\text {photon }}(x, y)$ is plotted using a linear false color scale as a function of the coordinates $x$ and $y$. For all $V_{\mathrm{SD}}$, we observe a dominating contribution of $\left|I_{\text {photon }}\right|$ at position I compared to $\left|I_{\text {photon }}\right|$ at position II of sample A [Fig. 1(c)]. We also detect $I_{\text {photon }}$ as a function of $V_{\mathrm{SD}}$ at positions I and II [Fig. 1(d)]. At position I, we observe a finite photocurrent $I_{0}$ at $V_{\mathrm{SD}}=0 \mathrm{~V}$ and a finite photovoltage $V_{0}$ at $I_{\text {photon }}=0$ A. Hereby, we confirm recent reports that a Schottky contact between a semiconductor nanowire and a metal pad can give rise to both a photocurrent and a photovoltage. ${ }^{8}$ In this process, the electron-hole pairs, which are locally created by photoexcitation in the $p$-doped GaAs nanowires, are separated due to the local built-in electric field at the Schottky contacts, and a maximum (minimum) photocurrent signal can be detected when the illuminated Schottky contact is reverse-biased (forward-biased). We note that at position I, $I_{\text {photon }}$ saturates at a source-drain voltage of
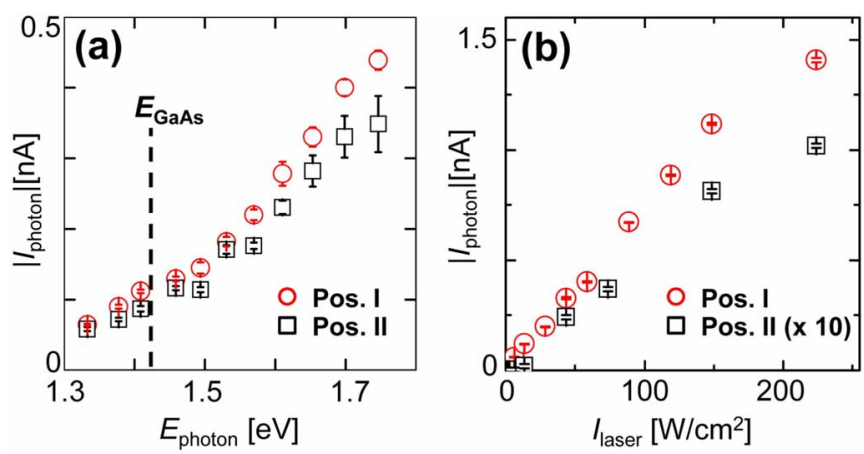

(c)

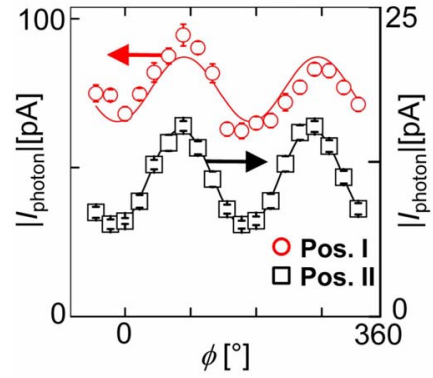

(d)

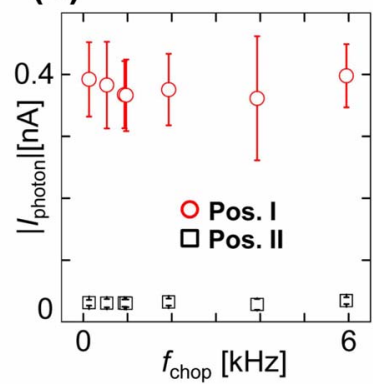

FIG. 2. (Color online) (a) $\left|I_{\text {photon }}\right|$ as a function of $E_{\text {photon }}$ for sample B for positions I (circles) and II (squares) as defined in Fig. 1(a) $\left(V_{\mathrm{SD}}=-1 \mathrm{~V}\right.$, $T_{\text {bath }} \sim 296 \mathrm{~K}, I_{\text {laser }} \sim 100 \mathrm{~W} / \mathrm{cm}^{2}$, and $f_{\text {chop }}=930 \mathrm{~Hz}$ ). (b) $\left|I_{\text {photon }}\right|$ as a function of the exciting laser intensity for sample A for positions I (circles) and II (squares) $\left(V_{\mathrm{SD}}=-1 \mathrm{~V}, T_{\text {bath }} \sim 296 \mathrm{~K}, f_{\text {chop }}=930 \mathrm{~Hz}\right.$, and $\lambda$ $=780 \mathrm{~nm}$ ). (c) $\left|I_{\text {photon }}\right|$ as a function of the angle $\phi$ between the linearly polarized laser field and the orientation of the nanowire for sample A for positions I (circles) and II (squares) $\left(V_{\mathrm{SD}}=+0.5 \mathrm{~V}, T_{\text {bath }} \sim 296 \mathrm{~K}, I_{\text {lase }}\right.$ $\sim 60 \mathrm{~W} / \mathrm{cm}^{2}, f_{\text {chop }}=930 \mathrm{~Hz}$, and $\lambda=800 \mathrm{~nm}$ ). (d) $\left|I_{\text {photon }}\right|$ as a function of $f_{\text {chop }}$ for sample A for positions I (circles) and II (squares) $\left(V_{\mathrm{SD}}=-1 \mathrm{~V}\right.$, $T_{\text {bath }} \sim 296 \mathrm{~K}, I_{\text {laser }} \sim 60 \mathrm{~W} / \mathrm{cm}^{2}$, and $\left.\lambda=780 \mathrm{~nm}\right)$.

$\left|V_{\text {sat }}\right| \sim 0.5 \mathrm{~V}$, and that both $V_{\text {sat }}$ and $I_{\text {photon }}\left(V_{\text {sat }}\right)$ depend on the laser intensity, as expected for a photocurrent process (data not shown). ${ }^{23}$ At position II, however, we observe only a negligible photocurrent and photovoltage signal at zero $V_{\mathrm{SD}}$ [Fig. 1(d)]. Here, we find that $I_{\text {photon }}$ linearly depends on $V_{\mathrm{SD}}$ of up to $\pm 1.5 \mathrm{~V}$ (data not shown). We interpret the observations at position II such that the optoelectronic response of the nanowire far away from the Schottky contacts is dominated by a photoconductance process, which will be discussed in the following.

Figure 2(a) depicts $\left|I_{\text {photon }}\right|$ of sample B as a function of $E_{\text {photon }}$ for a laser excitation at positions I and II, which are equally defined as in Fig. 1(a). The dashed line in Fig. 2(a) indicates the band gap energy of $E_{\mathrm{GaAs}} \approx 1.42 \mathrm{eV}$ of GaAs at room temperature. ${ }^{24}$ The noise level in the setup without laser excitation is below $\sim 2 \mathrm{pA}$. Since we detect a finite $\left|I_{\text {photon }}\right|$ for $E_{\text {photon }}<E_{\mathrm{GaAs}}$ for all positions on the nanowires, we conclude that there are parts of the nanowires, where an electric field gives rise to a Franz-Keldysh effect of the absorption energy ${ }^{25-27}$ and, alternatively, where surface states are optically excited. In the case of the Franz-Keldysh effect, we assume that the electric field can be attributed to the Fermi-level pinning of the oxidized $\{110\}$-facets of the $p$-doped GaAs with respect to the electrostatic potential of the holes in the center of the nanowires. ${ }^{28}$ We note that all data of the samples are taken in the linear response regime [Fig. 2(b)]. In this regime, the expected field strength can be estimated by the energy difference of $(0.4-0.5) \mathrm{eV}$ between the surface states and the Fermi-level within the nanowires ${ }^{28}$ and the lateral depletion length of typically $l_{\text {dep }}$ 
$\sim 20-30 \mathrm{~nm} .{ }^{29}$ The resulting field strength $F$ $\sim(0.4-0.5 \mathrm{eV}) /\left(e l_{\text {dep }}\right) \sim 10^{5} \mathrm{~V} / \mathrm{cm}$ is sufficient to ionize optically created excitons at the surface region of the nanowires. ${ }^{27,30}$ Separated charge carriers can contribute twofold to the photoconductance of the nanowires. On the one hand, the electrons can drift to the surface of the $p$-doped nanowires, acting as a local negative gating voltage (photogating effect or normal surface photovoltage). Hereby, the conductive part of the nanowire is widened. On the other hand, the holes increase the hole density within the nanowires (photodoping effect). ${ }^{31}$ Both effects can result in an optically increased conductance of the nanowire circuits. ${ }^{20,21}$ In addition, we note that the optical excitation of surface states for $E_{\text {photon }}<E_{\mathrm{GaAs}}$ can cause similar photogating and photodoping effects since the effective band bending favors a charge configuration with photon-generated electrons being localized on the surface of the nanowires, while the holes drift to the center of the nanowires.

The observed photoconductance and photocurrent signals depend on the polarization of the exciting laser field for both positions I and II [as depicted for sample A in Fig. 2(c)]. The corresponding signals follow a $\cos \phi$-like dependence, where $\phi$ is the angle between the wire and the light polarization. This observation is in agreement with recent reports that the nanowire geometry strongly affects the polarization of emitted or absorbed photons. ${ }^{2}$ We observe that the polarization ratio $\rho=\left(I_{\text {photon }}^{\|}-I_{\text {photon }}^{\perp}\right) /\left(I_{\text {photon }}^{\|}+I_{\text {photon }}^{\perp}\right)$ of $I_{\text {photon }}$ with parallel $\left(I_{\text {photon }}^{\|}\right)$and perpendicular $\left(I_{\text {photon }}^{\perp}\right)$ polarization orientations is larger at position II $(\sim 35 \%)$ than close to the Schottky contact of position I $(\sim 15 \%)$. Hereby, the data in Fig. 2(c) demonstrate that one can simply enhance $\rho$ by the described FIB-deposition technique since the technique allows suppressing the optoelectronic response of the nanowires due to the Schottky contacts by covering the contacts by an opaque carbon layer [position III in Figs. 1(a) and $1(\mathrm{~b})]$.

In order to test the response time of the metal-nanowiremetal photodetector, we measure $I_{\text {photon }}$ of the devices as a function of $f_{\text {chop }}$ [Fig. 2(d)]. For both positions I and II, we do not detect any dependence of $I_{\text {photon }}$ on $f_{\text {chop }}$ for frequencies between 0.1 and $6 \mathrm{kHz}$. This reveals that the effects causing the photocurrent (position I) and the photoconductance (position II) occur on a time scale shorter than $\sim(6 \mathrm{kHz})^{-1}=167 \mu \mathrm{s}$.

In summary, we present spatially and spectrally resolved optoelectronic measurements on freely suspended $p$-doped GaAs nanowires. We interpret the results in terms of the combination of a photogating and a photodoping effect, i.e. the consequence of the spatial separation of holes and electrons, in combination with a photocurrent effect, which is generated at the Schottky barriers between the GaAs nanowires and the metal electrodes. We demonstrate that both effects allow building a polarization dependent photodetector with a response time faster than $\sim 200 \mu \mathrm{s}$.

We gratefully acknowledge financial support by the DFG (Grant No. Ho 3324/4), the German Excellence Initiative via the "Nanosystems Initiative Munich (NIM)," Marie Curie Excellence Grant SENFED, and the Swiss National Science Foundation (Grant No. 2000021-121758/1) as well as the "Center for NanoScience" (CeNS) in Munich.
${ }^{2}$ J. Wang, M. S. Gudiksen, X. Duan, Y. Cui, and C. M. Lieber, Science 293, 1455 (2001)

${ }^{3}$ M. T. Bjork, B. J. Ohlsson, T. Sass, A. I. Persson, C. Thelander, M. H. Magnusson, K. Deppert, L. R. Wallenberg, and L. Samuelson, Appl. Phys. Lett. 80, 1058 (2002)

${ }^{4}$ S. De Franceschi, J. A. van Dam, E. P. A. M. Bakkers, L. F. Feiner, L. Gurevich, and L. P. Kouwenhoven, Appl. Phys. Lett. 83, 344 (2003).

${ }^{5}$ L. Samuelson, C. Thelander, M. T. Björk, M. Borgström, K. Deppert, K. A. Dick, A. E. Hansen, T. Mårtensson, N. Panev, A. I. Persson, W. Seifert N. Sköld, M. W. Larsson, and L. R. Wallenberg, Physica E 25, 313 (2004)

${ }^{6}$ O. Hayden, R. Agarwal, and C. M. Lieber, Nature Mater. 5, 352 (2006). ${ }^{7}$ E. D. Minot, F. Kelkensberg, M. van Kouwen, J. A. van Dam, L. P. Kouwenhoven, V. Zwiller, M. T. Borgström, O. Wunnicke, M. A. Verheijen, and E. P. A. M. Bakkers, Nano Lett. 7, 367 (2007).

${ }^{8}$ Y. Gu, E.-S. Kwak, J. L. Lensch, J. E. Allen, T. W. Odom, and L. J. Lauhon, Appl. Phys. Lett. 87, 043111 (2005).

${ }^{9}$ H. Kind, H. Yan, B. Messer, M. Law, and P. Yang, Adv. Mater. (Weinheim, Ger.) 14, 158 (2002).

${ }^{10}$ H. Pettersson, J. Tragardh, A. I. Persson, L. Landin, D. Hessman, and L. Samuelson, Nano Lett. 6, 229 (2006).

${ }^{11}$ T. Bryllert, L.-E. Wernersson, T. Löwgren, and L. Samuelson, Nanotechnology 17, S227 (2006).

${ }^{12}$ L. J. Lauhon, M. S. Gudiksen, C. L. Wang, and C. M. Lieber, Nature (London) 420, 57 (2002).

${ }^{13}$ K. A. Dick, K. Deppert, M. W. Larsson, T. Martensson, W. Seifert, L. R. Wallenberg, and L. Samuelson, Nature Mater. 3, 380 (2004).

${ }^{14}$ E. P. A. M. Bakkers, J. A. van Dam, S. de Franceschi, L. P. Kouwenhoven, M. Kaiser, M. Verheijen, H. Wondergem, and P. van der Sluis, Nature Mater. 3, 769 (2004)

${ }^{15}$ C. Colombo, D. Spirkoska, M. Frimmer, G. Abstreiter, and A. Fontcuberta i Morral, Phys. Rev. B 77, 155326 (2008).

${ }^{16}$ A. Fontcuberta i Morral, C. Colombo, J. Arbiol, J. R. Morante, and G. Abstreiter, Appl. Phys. Lett. 92, 063112 (2008).

${ }^{17}$ C. Colombo, M. Heiss, M. Grätzel, and A. Fontcuberta i Morral, Appl. Phys. Lett. 94, 173108 (2009).

${ }^{18}$ We utilize a FIB machine NVISION40 of ZEISS in combination with Kleindieck manipulators.

${ }^{19}$ Y. Peng, I. Luxmoore, M. D. Forster, A. G. Cullis, and B. J. Inkson, J. Phys.: Conf. Ser. 126, 012031 (2008).

${ }^{20}$ C. Rossler, K.-D. Hof, S. Manus, S. Ludwig, J. P. Kotthaus, J. Simon, A W. Holleitner, D. Schuh, and W. Wegscheider, Appl. Phys. Lett. 93, 071107 (2008)

${ }^{21}$ K.-D. Hof, C. Rossler, S. Manus, J. P. Kotthaus, A. W. Holleitner, D Schuh, and W. Wegscheider, Phys. Rev. B 78, 115325 (2008).

${ }^{22}$ Z. Y. Zhang, C. H. Jin, X. L. Liang, Q. Chen, and L.-M. Peng, Appl. Phys. Lett. 88, 073102 (2006).

${ }^{23}$ E. Beham, A. Zrenner, F. Findeis, M. Bichler, and G. Abstreiter, Appl Phys. Lett. 79, 2808 (2001).

${ }^{24} \mathrm{We}$ performed photoluminescence measurements on an ensemble of the $p$-doped nanowires located on top of a $\mathrm{SiO}_{2}$-chip at room temperature. The maximum of the photoluminescence at $E_{\mathrm{PL}} \sim 1.43 \mathrm{eV}$ agrees reasonably well with the band gap energy $E_{\mathrm{GaAs}}$ at room temperature.

${ }^{25}$ A. Schmeller, W. Hansen, J. P. Kotthaus, G. Trinkle, and G. Weimann, Appl. Phys. Lett. 64, 330 (1994).

${ }^{26}$ P. Baumgartner, C. Engel, G. Böhm, and G. Abstreiter, Appl. Phys. Lett. 70, 2876 (1997).

${ }^{27}$ A. Cavallini, L. Polenta, M. Rossi, T. Stoica, R. Calarco, R. J. Meijers, T Richter, and H. Lüth, Nano Lett. 7, 2166 (2007).

${ }^{28}$ W. E. Spicer, P. W. Chye, C. M. Garner, I. Lindau, and P. Pianetta, Surf. Sci. 86, 763 (1979)

${ }^{29}$ S. P. Riege, T. Kurth, F. Runkel, D. Heitmann, and K. Eberl, Appl. Phys. Lett. 70, 111 (1997).

${ }^{30}$ A. Gärtner, L. Prechtel, D. Schuh, A. W. Holleitner, and J. P. Kotthaus, Phys. Rev. B 76, 085304 (2007).

${ }^{31}$ The magnitude of $\left|I_{\text {photon }}\right| \sim 20 \mathrm{pA}[$ Fig. 1(d)] suggests a relative change in the number of holes per laser chopping cycle $\Delta N_{h} / N_{h} \propto \Delta E_{F} / E_{F}$ $\propto\left|I_{\text {photon }}\right| /\left|I_{\mathrm{SD}}\right| \cong 10^{-3}$, with $E_{F}$ the Fermi energy. The total number of holes in the active layer can be estimated to be $N_{h}=V_{\text {active }} n_{s} \cong 10^{5}$, with $n_{s}$ the hole density and $V_{\text {active }}$ the active volume of the nanowire. The corresponding number of excess holes $\Delta N_{h}=10^{2}$ is smaller than the number $N_{\text {photon }}$ of absorbed photons per cycle, which can be estimated as $\quad N_{\text {photon }}=I_{\text {laser }} A \alpha d /\left(E_{\text {photon }} f_{\text {chop }}\right)=30 \mu \mathrm{W} / \mathrm{cm}^{2} \times\left(2.8 \times 10^{-9} \mathrm{~cm}^{2}\right)$ $\times 0.014 /(1.7 \mathrm{eV} \times 930 \mathrm{~Hz}) \approx 10^{5}$, with $A$ the illuminated area of the wire, $d$ its thickness, and $\alpha$ the absorption coefficient of GaAs, thus enabling the discussed photodoping effect. 